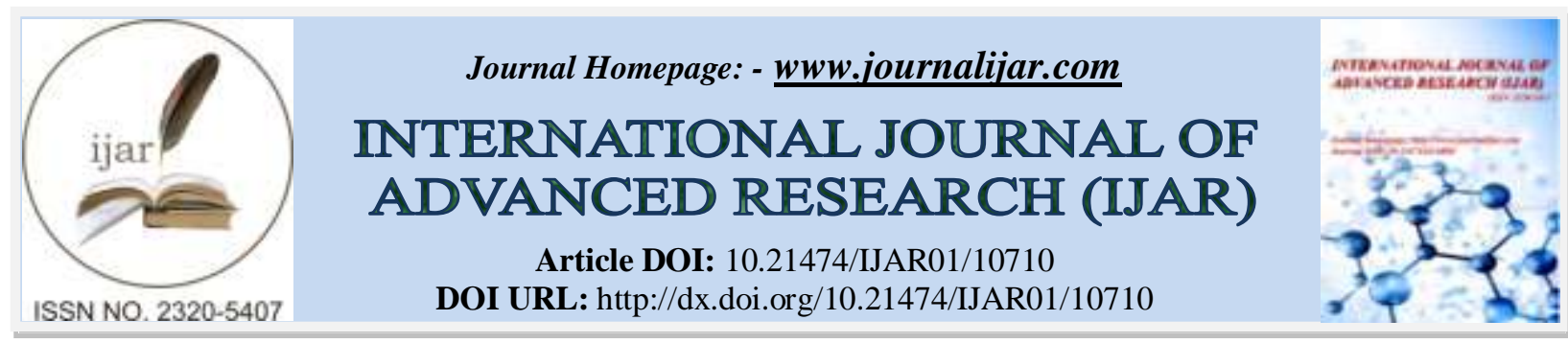

RESEARCH ARTICLE

\title{
CARDIOVASCULAR RISK PROFILE AND RISK STRATIFICATION OF THE HYPERTENSIVE POPULATION: A CROSS-SECTIONAL ANALYSIS OF A 200 PATIENT DATABASE
}

A. Chetoui, R. Chniber, I. Hindi, A. Hadari, A. Zbitou, L. Bendriss and A. Khatouri Departement of Cardiology, Avicenne Military Hospital, Marrakech.

\section{Manuscript Info} ………………....

Manuscript History

Received: 22 January 2020

Final Accepted: 25 February 2020

Published: March 2020

Key words:-

Cardiovascular Risk - HTA

\section{Abstract}

Hypertension is a major cause of death worldwide; it is one of the most important cardiovascular risk factors. Our study aim to determine the incidence of main factors of cardiovascular risk at our patients, according to a cross-sectional study, made in the cardiology department at the military hospital Avicenne of Marrakesh, over a period of one year: from January 2017to December 2017. The study concerned a sample of 200 hypertensive patients. The mean age of the population is $58,86 \pm 9,93$ years, women are affected more than the men with a sex ratio of $0,71,42 \%$ of the patients had DM2, $19,5 \%$ were smokers, and $34 \%$ suffer from hypercholesterol level, $49 \%$ are obese with the middleweight of $82 \pm 12,9 \mathrm{~kg}$, the average waist measurement is 88,85 $\pm 12,65 \mathrm{~cm}$, the systolic average blood pressure is $150,86 \pm 20,1 \mathrm{mmHg}$, the diastolique blood pressure average is $84,9 \pm 11,95 \mathrm{mmHg}, 24 \%$ of the EKG were abnormal: 18,8\% showed LAH, 13,5\% secondary repolarization abnormalities, 10,4\% LVH. In conclusion, $60 \%$ of the participants were considered very high risk, $37,5 \%$ had medium risk, and $2,5 \%$ lowrisk.

Copy Right, IJAR, 2020,. All rights reserved.

\section{Introduction:-}

High blood pressure, affects nearly 600 million people worldwide [1], it is the leading cause of death in most developed countries, and secondarily in developing countries. In Morocco, which is currently undergoing an epidemiological transition phase, high blood pressure constitutes a real public health problem and represents one of the main risk factors for cardiovascular diseases (33\% is the global prevalence of hypertension in Morocco) [ 2]. In addition, it is one of the main risk factors for cardiovascular diseases (CVD), which are characterized by multiple risk factors that are often entangled [3].

Therefore, the recommendations of the World Health Organization (WHO) and the International Society of Hypertension (ISH) [4] emphasized on the importance of strictassessment of cardio-vascular risk before undergoing a high blood pressure treatment, based not only on the level of blood pressure but also on the number of cardiovascular risk factors and the existence of target organs damages. Thus, the cardiovascular risk is a multidimensional entity in which several interacting parameters take part, and whose consideration in an integrated approach is mandatory for a preventive strategy.

Corresponding Author:- A. Chetoui

Address:- Departement of Cardiology, Avicenne Military Hospital, Marrakech. 
Our study aims to estimate the prevalence of cardiovascular risk factors in hypertensive patients, followed in the cardiology department at the Avicenne Military Hospital, and the comparison between the results found in our study with other studies.

\section{Material and Methods:-}

This is a prospective study, with a sample of 200 hypertensive patients who were randomly selected from patients consulting the cardiology department at the Avicenne military hospital in Marrakech, over a period of one year from January 2017 to December 2017. Data collection was conducted using a questionnaire. The variables studied included anamnestic data, pathological antecedents, anthropometric measures (weight, height, waist circumference), and blood tests. A clinical examination with blood pressure and anthropometric measurements were performed on site. Blood pressure was measured using a VAQUEZ type blood pressure monitor, three times at 10 minute intervals. High blood pressure was defined as systolic blood pressure greater or equal to $140 \mathrm{mmHg}$ and / or diastolic blood pressure greater or equal to $90 \mathrm{mmHg}$. Measurement of weight was performed on lightly dressed subjects on a calibrated Seca scale. The height was measured with barefooted subjects standing the head and the back in contact with the board. Waist circumference was measured with a tape measure on a horizontal plane, at a point midway between the last rib and the iliac crest during exhaling. Abdominal obesity was defined as waist circumference greater than 94 centimetres in men and more than 80 centimetres in women, the body mass index $(\mathrm{BMI}=$ weight $/$ height2) was calculated with the weight in $\mathrm{kg}$ and the height in centimetres. Obesity has been defined by a BMI greater than or equal to $30 \mathrm{~kg} / \mathrm{m} 2$. Diabetes was retained in the presence of fasting glucose greater than or equal to $1.26 \mathrm{~g} / \mathrm{l}$, dyslipidaemia was defined as a total cholesterol level greater than or equal to $2.4 \mathrm{~g} / \mathrm{l}$, and / or a triglyceride level greater than or equal to $2 \mathrm{~g} / 1$. Anyone who does not exercise at least 30 minutes of moderate intensity exercise every day, which is equivalent to half an hour of brisk walking, is considered as sedentary. The risk factors selected were: male sex, age greater than 50 years in men and 60 years in women, history of high blood pressure in parents, smoking, dyslipidaemia, diabetes, obesity, target organ damage (heart and vessels, brain, eye, kidney). We used the classification of the National Agency for Accreditation and Evaluation in Health (ANAES) [5]. Therefore, we rated our patients as low, medium, high and very high risk.

\section{Results:-}

The sample size was 200subjects, the sex ratio was 0.71 in favor of women. The average age of participants is 58.86 \pm 9.93 years, diabetes was the most important risk factor. Table I summarizes the general characteristics of the studied population.

\begin{tabular}{|l|l|}
\hline Parameter & Population \\
\hline Mean age (years) & $58.86+/-9.93$ \\
\hline SBP $(\mathrm{mmHg})$ & $150.86+/-20.1$ \\
\hline DBP $(\mathrm{mmHg})$ & $84.9+/-11.95$ \\
\hline Diabetes & $84(42 \%)$ \\
\hline Dyslipidaemia & $46(26 \%)$ \\
\hline Smoking & $39(19.5 \%)$ \\
\hline Overweight & $91(45.5)$ \\
\hline Complication & $9(4.5 \%)$ \\
\hline
\end{tabular}

The mean systolic BP was $150.86 \pm 20.1$ and the diastolic BP was $84.9 \pm 11.95 \mathrm{mmHg}$. High blood pressure grade II represents $36.5 \%, 33 \%$ have a grade I, and $29 \%$ of the patients have grade III. The prevalence of diabetes is estimated at $42 \%$, only one patient had type 1 diabetes, with a higher prevalence among women. Overweight (BMI $25-30 \mathrm{~kg} / \mathrm{m}^{2}$ ) is found in $36.5 \%$ of the studied population; obesity, defined by a BMI $\geq 30 \mathrm{~kg} / \mathrm{m}^{2}$ is found in $49 \%$ of the population. Abdominal obesity is observed in $62.4 \%$ of women, and $44.4 \%$ of men. Smoking was strictly limited to the male population, $19.5 \%$ is the overall prevalence. Blood tests showed $36 \%$ of the patients had a fasting blood glucose $\geq 1,26 \mathrm{~g} / 1$ is, among these patients $91,7 \%$ have a postprandial glucose level higher than $2 \mathrm{~g} / \mathrm{l}$. Hypercholesterolemia was found in $34 \%$ of subjects, and hypertriglyceridemia was found in $16.5 \%$ of the total population. EKG were normal in $64 \%$ of patients, $24 \%$ showed abnormalities: $18.8 \%$ have left atrial hypertrophy (LAH), $13.5 \%$ repolarization disorder, 10, 4\% left ventricular hypertrophy (LVH). Dietary and dietary measures (HDM) were applied in all patients, antihypertensive monotherapy in 55.5\% of cases, dual therapy in $42 \%$ of patients, and triple therapy in $2.5 \%$ of patients. The distribution of patients according to the cardiovascular risk according to the stratification of ANAES followed was: 120 (60\%) patients were considered as very high risk, 75 $(37.5 \%)$ average risk, and $5(2.5 \%)$ low risk. 


\section{Discussion:-}

Cardio-vascular risk is a complex phenomenon involving many factors; this is why it can not be determined in terms of causes, but by using notions of risk factors and indicators [6]. One of the most important risk factors that increase the risk of coronary heart disease is diabetes, $42 \%$ of our patients had diabetes, with a higher prevalence in women (56\% vs 44\%). These results are close to those observed in Algeria (54.8\%) [7], and higher than those observed in Togo (4.5\%) [8]. Half of these diabetic patients are overweight and $20.2 \%$ have dyslipidaemia. Considering the majored risk of this combination, two different categories should be set: kidney disease and cardiovascular disease. Our study showed other frequent and associated risk factors: dyslipidaemia (23\%), overweight (45\%), and smoking $(19.5 \%)$. The simultaneous presence of hypertension and dyslipidaemia increases cardiovascular risk and is frequently associated to insulin resistance. The link between hypercholesterolemia and atherosclerosis disease is well established, mainly for coronary heart disease. The elevation of total cholesterol and LDL-cholesterol is associated with an increased risk of coronary heart disease in a curvilinear way and has been established in inter and intra-population epidemiological studies. 34\% of our patients suffer from hypercholesterolemia. The average weight is $82+/-12.9 \mathrm{~kg}, 14.5 \%$ of patients have a BMI <25 kg / m2, 36.5\% between $25-30 \mathrm{~kg} / \mathrm{m} 2$ and $49 \%>$ to $30 \mathrm{~kg} /$ $\mathrm{m} 2$, therefore, half of the patients are obese. Numerous studies have shown that there is a strong relationship between body mass index (BMI) and systolic and diastolic blood pressure. The anatomical distribution of body fat plays an important role in numerous diseases associated to obesity. Studies have shown that visceral adipose tissue loss measured by tomography is closely correlated with a decrease in systolic and diastolic blood pressure. In our study, almost half of the patients who have abdominal obesity have diabetes. Among the modifiable risk factors, we findsmoking. $44.6 \%$ of male patients of our study are smokers, and no woman was smoking. For the same blood pressure level, smoking patients have more frequent target organ damages than non-smokers and can be classified as high cardiovascular risk group. Moreover, it is well established that cardiovascular risk increases with age. The NAAHE (National Agency for Accreditation of Health Evaluation) considers age as a risk factor from 50 years for men and 60 years for women. In our study the average age was $58.86 \pm 9.93$ years, among 117 men, $61 \%$ are $>50$ years old, and among 70 women $27 \%$ are over 60 years old. As far as sex is concerned, more men have coronary atherosclerosis than women. In our study $58.5 \%$ of the patients are women, $41.5 \%$ are men with a sex ratio $\mathrm{H} / \mathrm{F}$ of 0.71 .

\section{Conclusion:-}

Cardiovascular risk factors are most often asymptomatic and must be detected (high blood pressure). Simple elements (age, overweight, high blood pressure) must lead to complementary biological investigations (glycemia, lipid balance, electrocardiogram, etc.). Coexistence of multiple risk factors should always be sake.

\section{Références:-}

1. Chobanian A. Control of Hypertension - An Important National Priority. N Engl J Med 2001;345:534-5.

2. Tazi MA, Abir-Khalil S, Chaouki N. Prevalence of the main cardiovascular risk factors in Morocco: result of a national survey 2000. Journal of hypertension 2003; 21:897-903.

3. Erica J, Lawrence E, Peter R. Cardiovascular and coronary risk estimation in hypertension management. Heart 2002;88:306-12.

4. Guidelines SubComitté. 1999 World Health Organisation-Internationnal Society of Hypertension Guidelines for the management of Hypertension. Journal of Hypertension 1999;17:151-83.

5. Agencenationaled'accréditationetd'évaluation en santé (ANAES). Prise en charge des patientsadultesatteintsd'hypertensionartérielleessentielle. PARIS ANAES 2000.

6. Furmeron $\mathrm{C}$ et al. Aspects cliniques, exploration et prise en charge de l'hypertensionartérielle. Encycl Med Chir, Cardiologie-angéiologie 1995;11-301-B-10,8P.

7. Sadaoui L, Batouche D, Berachedi W, Achou S, Kaddous A. Hypertension artérielle et risque cardiovasculaire.10.1016/j.nephro.2011.07.195.

8. Damorou F, Pessinaba S, Tcherou T, YayehdNdassa S, Soussou B. Hypertension artérielle du sujet noir âgé de 50 ans et plus à Lomé : aspects épidémiologiques et évaluation du risquecardiovasculaire. Annales de Cardiologie et d'Angéiologie 2011;60:61-6. 